\title{
Correction to: Epigenomic, genomic, and transcriptomic landscape of schwannomatosis
}

\author{
Sheila Mansouri ${ }^{1}$. Suganth Suppiah ${ }^{1} \cdot$ Yasin Mamatjan ${ }^{1} \cdot$ Irene Paganini $^{2} \cdot$ Jeffrey C. Liu ${ }^{1} \cdot$ Shirin Karimi $^{1}$. \\ Vikas Patil ${ }^{1}$ - Farshad Nassiri ${ }^{1}$. Olivia Singh ${ }^{1}$ - Yogi Sundaravadanam ${ }^{3}$. Prisni Rath ${ }^{3} \cdot$ Roberta Sestini $^{2}$. \\ Francesca Gensini ${ }^{2}$. Sameer Agnihotri ${ }^{4}$. Jaishri Blakeley ${ }^{5} \cdot$ Kimberly Ostrow $^{5} \cdot$ David Largaespada $^{6}$. \\ Scott R. Plotkin ${ }^{7} \cdot$ Anat Stemmer-Rachamimov $^{7} \cdot$ Marcela Maria Ferrer $^{8} \cdot$ Trevor J. Pugh $^{3} \cdot$ Kenneth D. Aldape $^{9}$. \\ Laura Papi ${ }^{2} \cdot$ Gelareh Zadeh ${ }^{1,10,11}$
}

Published online: 28 October 2020

(c) Springer-Verlag GmbH Germany, part of Springer Nature 2020

\section{Correction to: Acta Neuropathologica https://doi.org/10.1007/s00401-020-02230-x}

In the original publication, corresponding author information missed indicating that Dr. Zadeh and Dr. Papi are cocorresponding and co-senior authors of this manuscript.

Author contributions LP and GZ conceived the entire project. Together with SM they designed, performed and analyzed the majority of the experiments in this study, prepared figures, and wrote the manuscript. SM, YM, SS, and IP collected and analyzed data. YM, JL, VP, YS, and PR performed bioinformatics data analysis. KA, ASR, and SK performed pathology review of the cases. RS and FG helped with patient characterization. MMF and LP provided data and samples. SA, SK, DL, FN, OS, KO, SRP, and JB provided technical assistance, data

Gelareh Zadehand Laura Papi are co-senior authors of this manuscript.

The original article can be found online at https://doi.org/10.1007/ s00401-020-02230-x.

Laura Papi

laura.papi@unifi.it

$\triangle$ Gelareh Zadeh

Gelareh.zadeh@uhn.ca

1 Princess Margaret Cancer Center and MacFeeters-Hamilton Center for Neuro-Oncology Research, University Health Network, Wilkins Family Chair in Brain Tumor Research, 14-701 PMCRT, 101 College St, Toronto, ON M5G 1L7, Canada

2 The Department of Experimental and Clinical, Medical Genetics Unit, Biomedical Sciences "Mario Serio", University of Florence, Florence, Italy

3 Ontario Institute for Cancer Research, Toronto, ON, Canada

4 Department of Neurological Surgery, Children's Hospital, University of Pittsburgh, Pittsburgh, PA, USA interpretation, and reviewed the manuscript. TJP provided computational expertise.

Publisher's Note Springer Nature remains neutral with regard to jurisdictional claims in published maps and institutional affiliations.

5 Johns Hopkins University, Baltimore, MD, USA

6 Department of Paediatrics, University of MN, Minneapolis, USA

7 Department of Pathology, Massachusetts General Hospital, Boston, MA, USA

8 División de Neurocirugía and División Genética, Hospital de Clínicas “José de San Martín”, Universidad de Buenos Aires, Buenos Aires, Argentina

9 Laboratory of Pathology, Center for Cancer Research, National Cancer Institute, Bethesda, MD, USA

10 Division of Neurosurgery, Toronto Western Hospital, Toronto, Canada

11 Krembil Brain Institute, Toronto, Canada 